\title{
Association of Long-Chain Noncoding RNA H19 and MEG3 Gene Polymorphisms and Their Interaction with Risk of Osteoarthritis in a Chinese Han Population
}

\author{
Huang Wang, Jian Li, ${ }^{2}$ Ye Cheng, ${ }^{3}$ and Jun Yao ${ }^{1}$
}

\begin{abstract}
Objective: To analyze the association of single nucleotide polymorphisms (SNPs) in the long-chain noncoding RNAs (lncRNAs) H19 and maternal expressed gene 3 (MEG3) with the risk of osteoarthritis (OA) in a Chinese Han population.

Methods: A total of 230 patients with OA and 230 control subjects were recruited. Sanger sequencing was used to analyze the genotypes of the H19 gene rs 217727 and rs3741219 loci and the MEG3 gene rs 7158663 locus. Quantitative real-time reverse transcription-polymerase chain reaction was used to determine the levels of the microRNAs (miRNA) hsa-miR-4804-5p, hsa-miR-8071, hsa-miR-8072, hsa-miR-3960, hsa-miR-4307, and hsamiR-1265 in the plasma.

Results: The risk of OA was significantly increased by the A allele of the rs217727 locus of $H 19$ (odds ratio $=1.27,95 \%$ confidence interval [CI]: $1.09-1.42, p=0.001)$. The $H 19$ gene rs3741219 locus SNP had no significant correlation with the risk of OA $(p>0.05)$. The risk of OA in subjects carrying the rs7158663 locus A allele of $M E G 3$ was 1.32 times higher compared with those carrying the $\mathrm{G}$ allele (95\% CI: $1.16-1.50, p<0.01)$. SNP rs217727 of $H 19$ was associated with the plasma levels of lncRNA H19, hsa-miR-4804-5p, hsa-miR-8071, hsa-miR-8072, and hsa-miR-3960, and SNP rs7158663 in MEG3 was associated with the plasma levels of lncRNA MEG3, hsa-miR-4307, and hsa-miR-1265.

Conclusion: Specific SNPs of the H19 rs217727 and MEG3 rs7158663 loci are associated with the risk of OA. The possible underlying molecular mechanisms, which remain to be confirmed, are their likely influence on the expression levels of the lncRNA H19 and MEG3, which in turn alters the expression level of their target miRNAs.
\end{abstract}

Keywords: long-chain noncoding RNA, osteoarthritis, single nucleotide polymorphism, H19, MEG3

\section{Introduction}

O STEOARThritis (OA) Is a common type of delayed degenerative arthritis. Its main features include the progressive degeneration of articular cartilage, narrowing of the articular space, subchondral sclerosis, and the formation of osteophytes at the joint margins. These symptoms lead to chronic arthralgia and movement limitation (Lane et al., 2017; Kim et al., 2019; Naili et al., 2019). Common symptoms of OA include joint swelling, stiffness, arthralgia, and decreased movement (Herman et al., 2015; Schuring et al., 2017).

Little is known about the relative importance of etiology or bone remodeling and cartilage degradation in OA. Recently, the incidence of primary knee OA was reported to be as high as $19.4 \%$ in a Chinese population over 60 years of age (Xiang and Dai, 2009). In addition to factors, such as age, gender, body mass index (BMI), ethnicity, and trauma, many genetic factors are closely linked to the onset of OA (Deane et al., 2017; Traylor et al., 2017). Hence, to develop OA drugs, it may be necessary to screen the susceptibility genes of OA and study their relationship with the risk of OA and its molecular mechanisms.

Noncoding RNAs (ncRNAs) are a class of RNA molecules that do not encode proteins. They include long-chain ncRNAs (lncRNAs) and microRNAs (miRNAs). LncRNAs play an important role in the development of bone and cartilage, suggesting that lncRNAs have a potential role as molecular targets to diagnose and treat OA (Ponting et al., 2009; Fu et al., 2015). The human lncRNA gene H19 is

\footnotetext{
${ }^{1}$ Department of Orthopedics, Hangzhou Dingqiao Hospital (Hangzhou Hospital of Traditional Chinese Medicine, Dingqiao District), Hangzhou, China.

${ }^{2}$ Department of Spinal Surgery, Jinan Central Hospital Affiliated to Shandong University, Jinan, China.

${ }^{3}$ Department of Orthopedics, The Second Affiliated Hospital of Zhejiang Chinese Medical University, Hangzhou, China.
} 
located on chromosome $11 \mathrm{p} 15.5$. Previous research on IncRNA H19 has concentrated on its possible roles in the occurrence and development of tumors. More recently, evidence was presented indicating that lncRNA H19 mediates multiple differentiation processes of mesenchymal stem cells, regeneration of the musculoskeletal system, and the process of bone metabolism (Liao et al., 2017). The lncRNA gene maternal expressed gene 3 (MEG3) is located on chromosome $14 q 32.3$. Inactivation of $M E G 3$ leads to angiogenesis, which might be one of the mechanisms that promote the progression of OA. MEG3 knockdown leads to the progression of OA, probably through the miRNA (miR)16/SMAD7 (SMAD family member 7) axis (Xu and $\mathrm{Xu}$, 2017).

The $H 19$ rs217727 and rs3741219 single nucleotide polymorphism (SNP) loci and the MEG3 rs7158663 SNP locus are located at the miRNA-binding sites of these lncRNAs (Ghaedi et al., 2018). We speculated that the SNPs might affect the binding efficiency of the lncRNAs to miRNAs and, in turn, alter the regulation of the miRNAs by the IncRNAs. Li and Niu (2019) showed that the rs217727 locus of the $H 19$ gene is associated with risk of bladder cancer. Although there is no direct evidence, the results of this study suggest that the mechanism of this correlation is related to the mutation affecting the function of lncRNA H19 in bladder cancer. Although there is no evidence to support that the rs3741219 locus is associated with the risk of OA, similar to the rs 217727 locus, the rs3741219 locus is also located at the binding site of IncRNA H19 and miRNA, and the role of its genetic variation on the function of lncRNA H19 is not clear. Ghaedi et al. (2018) showed that there is a significant correlation between $M E G 3$ rs7158663-AA genotype and type 2 diabetes susceptibility. Therefore, we selected these SNP sites and designed a case-control study to analyze the H19 gene rs217727 and rs3741219 loci SNPs and the MEG3 gene rs7158663 locus SNP in 230 patients with OA and 230 control individuals, as well as their associations with the risk of OA.

\section{Materials and Methods}

\section{Subjects}

From May 2016 to January 2019, 230 Chinese Han patients with knee OA were recruited in this study according to the diagnostic and exclusion criteria of OA. The patients were $32-85$ years of age (mean $=62.36 \pm 9.66$ years). The diagnostic criteria for OA were based on the symptom criteria of the American College of Rheumatology and included at least one radiographic sign of knee OA (Kellgren and Lawrence $[\mathrm{K} / \mathrm{L}]$ grade $\geq 2$ ) (Kellgren and Lawrence, 1957). A group of 230 healthy Chinese Han subjects who were matched by age ( \pm 5 years) and gender (men $\pm 5 \%$ ) with the case group was included as healthy controls. The severity of OA was evaluated according to the $\mathrm{K} / \mathrm{L}$ classification. The X-ray films of the knee joints of the control group showed a K/L score $<2$. The controls were between 36 and 87 years of age $($ mean $=$ $62.29 \pm 13.21$ years). The exclusion criteria were other types of arthritis, previous history of knee surgery, metabolic bone disease, rheumatoid arthritis, diabetes, and hypertension. This study was approved by the Medical Ethics Committee of the Hangzhou Dingqiao Hospital (Hangzhou Hospital of Traditional Chinese Medicine, Dingqiao District). All sub- jects provided signed informed consent. The recruitment was performed in accordance with the World Medical Association Declaration of Helsinki.

\section{Genotype analysis of $\mathrm{H} 19$ and MEG3 genes}

Five milliliters of venous blood was extracted from all subjects. The plasma was isolated and EDTA-K2 was used for anticoagulation. The genomic DNA of each subject was purified from peripheral blood mononuclear cells using the model 61104 QIAamp DSP DNA Blood Mini Kit (Qiagen, Hilden, Germany) and stored in a refrigerator at $-20^{\circ} \mathrm{C}$. Polymerase chain reaction (PCR) primers were designed according to the sequence information of the $H 19$ gene loci rs217727 and rs3741219 and the MEG3 gene locus rs7158663 in the National Center for Biotechnology Information database. The primer sequences for $H 19$ locus rs217727 were: 5'-GCT CAG CTC TGG GAT GAT GT-3' (forward) and 5'-ACT CCA TCT TCA TGG CCA CC-3' (reverse). The primer sequences for locus rs3741219 were: 5'-AGT CAC CCG GCC CAG AT-3' (forward) and 5'-AGT ACG AGT GTG CGT GAG TG-3' (reverse). The primer sequences for MEG3 locus rs7158663 were: 5'-GCT GAG ATT CGG GAT AGG GT-3' (forward) and 5'-GTA GGG AGC ACG TGA AGG AT-3' (reverse). The primers were synthesized by Sangon Biotech Co. (Shanghai, China). The extracted genomic DNA was used as a template for PCR. The reaction system comprised $20 \mu \mathrm{L}$ and included $50 \mathrm{ng}$ of genomic DNA, $2 \mu \mathrm{L}$ of $10 \times$ PCR buffer, $1.5 \mu \mathrm{L}$ of $25 \mathrm{mmol} / \mathrm{L}$ $\mathrm{Mg}^{2+}, 1 \mu \mathrm{L}$ of $10 \mu \mathrm{mol} / \mathrm{L}$ dNTPs, $1 \mu \mathrm{L}$ of $10 \mathrm{pmol} / \mathrm{L}$ of each primer, $1 \mathrm{U}$ of DNA polymerase, and $20 \mu \mathrm{L}$ of sterile water. The PCR conditions were: predenaturation at $95^{\circ} \mathrm{C}$ for $5 \mathrm{~min}$, 30 cycles of denaturation at $94^{\circ} \mathrm{C}$ for $30 \mathrm{~s}$, annealing at $60^{\circ} \mathrm{C}$ for $30 \mathrm{~s}$, extension at $72^{\circ} \mathrm{C}$ for $30 \mathrm{~s}$, and a final extension at $72^{\circ} \mathrm{C}$ for $5 \mathrm{~min}$. The genotypes of the $H 19$ loci rs 217727 and rs3741219 and the MEG3 locus rs7158663 locus were analyzed using Sanger sequencing. Twenty percent of the samples were randomly selected for repeated detection to verify the accuracy of the sequencing results.

\section{Quantitative real-time reverse transcription-PCR}

Total RNA was extracted from plasma using the TRIzol reagent (Invitrogen, Carlsbad, CA). The extracted RNA was reverse transcribed into complementary DNA (cDNA) using PrimeScript RT Master Mix (TaKaRa Bio, Dalian, China). The cDNA was subjected to quantitative PCR (qPCR) performed using the PrimeScript RT Kit and SYBR Premix Ex Taq (Ta$\mathrm{KaRa} \mathrm{Bio}$ ) according to the manufacturer's instructions. The transcription of the glyceraldehyde-3-phosphate dehydrogenase $(G A P D H)$ gene was used as an internal control. The primers used were synthesized by Sangon Biotech Co. The primer sequences for hsa-miR-4804-5p were 5'-GTT AGC ACC TTC GCC GTA G-3' (forward) and 5'-AAG CTA AAG AAA AAG AGA CCG-3' (reverse). The primer sequences for hsa-miR-8071 were 5'-AAC ATT CAA CGC TGT CGG TG$3^{\prime}$ (forward) and 5'-AGC CAT AGG GTA CAA TCA ACG G-3' (reverse). The primer sequences for hsa-miR-8072 were 5'-GTC AAG ATG GCG GCG GG-3' (forward) and 5'-GAC TGG AGC GGA GGC G-3' (reverse). The primer sequences for hsa-miR-3960 were 5'-GTC AAG ATG GCG GCG GG-3' (forward) and 5'-GAC TGG AGC GGA GGC G-3' (reverse). The primer sequences for hsa-miR-4307 were 5'-GTC AAG 
Table 1. Comparison of the Basic Characteristics Between Patients With Osteoarthritis AND CONTROLS

\begin{tabular}{lccc}
\hline & $\begin{array}{c}\text { OA group } \\
(\mathrm{n}=230), \mathrm{n}(\%)\end{array}$ & $\begin{array}{c}\text { Control group } \\
(\mathrm{n}=230), \mathrm{n}(\%)\end{array}$ & $\mathrm{p}$ \\
\hline Age (years) & $62.36 \pm 9.66$ & $62.29 \pm 13.21$ & 0.95 \\
Gender & & & 0.92 \\
$\quad$ Men & $89(38.70)$ & $88(38.26)$ & \\
$\quad$ Women & $141(61.30)$ & $142(61.74)$ & \\
BMI $\left(\mathrm{kg} / \mathrm{m}^{2}\right)$ & $25.91 \pm 2.37$ & $25.37 \pm 4.12$ & 0.09 \\
Kellgren-Lawrence grading & & \\
2 & $75(32.61)$ & & \\
3 & $84(36.52)$ & & \\
4 & $71(30.87)$ & & \\
\hline
\end{tabular}

BMI, body mass index; OA, osteoarthritis.

ATG GCG GCG GG-3' (forward) and 5'-GAC TGG AGC GGA GGC G-3' (reverse). The primer sequences for hsa-miR1265 were $5^{\prime}$-GTC AAG ATG GCG GCG GG-3' (forward) and 5'-GAC TGG AGC GGA GGC G-3' (reverse). The primer sequences for GAPDH were 5'-GTC AAC GGA TTT GGT CTG TAT T-3' (forward) and 5'-AGT CTT CTG GGT GGC AGT GAT-3' (reverse). The conditions for qPCR were: $95^{\circ} \mathrm{C}$ for $30 \mathrm{~s}, 40$ cycles of $95^{\circ} \mathrm{C}$ for $5 \mathrm{~s}$, and $60^{\circ} \mathrm{C}$ for $34 \mathrm{~s}$. The $2^{-}$ $\triangle \Delta \mathrm{CT}$ method was used to calculate the fold change in expression of miRNAs relative to that of GAPDH.

\section{Statistical analyses}

The statistical analyses were performed using SPSS software (version 22.0; SPSS, Inc., Chicago, IL). Continuous variables are expressed as the mean \pm standard deviation, and statistical analyses between groups were performed using a $t$-test. The categorical variables are expressed as $n(\%)$ and the statistical analyses between groups were performed using a $\chi^{2}$ test. The $\chi^{2}$ test was used to analyze whether the genotype distribution was consistent with the Hardy-Weinberg equilibrium. The association between H19 SNPs and MEG3 SNPs with the risk of OA was estimated using unconditional logistic regression analysis to calculate odds ratios (ORs) and 95\% confidence intervals (CIs) adjusting for age, gender, and BMI by Bonferroni's method. Pearson correlation was performed to analyze the correlation between lncRNAs and miRNAs. All tests were two-tailed, with $p<0.05$ indicating a significant difference.

\section{Results}

\section{Basic characteristics of subjects}

The basic characteristics of the 230 patients with OA and the 230 controls are shown in Table 1 . There were no significant differences in age, gender, and BMI between the two groups $(p>0.05)$. The $\mathrm{K} / \mathrm{L}$ grading results of the 230 patients with OA showed that $32.61 \%$ were at grade $2,36.52 \%$ were at grade 3 , and $30.87 \%$ were at grade 4 .

Table 2. Association of Genotype and Allele Frequency of $H 19$ Rs217727 and rs3741219 Loci AND $M E G 3$ RS7158663 LOCUS WITH THE RISK OF OSTEOARTHRITIS

\begin{tabular}{|c|c|c|c|c|c|}
\hline & $O A(\mathrm{n}=230), \mathrm{n}(\%)$ & Control $(\mathrm{n}=230), \mathrm{n}(\%)$ & $H W E \mathrm{p}$ & OR $(95 \% C I)^{*}$ & $\mathrm{p}^{*}$ \\
\hline \multicolumn{6}{|l|}{ rs217727 } \\
\hline GG & $84(36.52)$ & $94(40.87)$ & 0.84 & 1.00 (reference) & \\
\hline GA & $75(32.61)$ & $105(45.65)$ & & $0.88(0.69-1.13)$ & 0.34 \\
\hline $\mathrm{AA}$ & $71(30.87)$ & $31(13.48)$ & & $1.48(1.19-1.79)$ & $<0.01$ \\
\hline Additive & & & & $1.06(0.88-1.29)$ & 0.58 \\
\hline Dominant & & & & $1.10(0.90-1.35)$ & 0.39 \\
\hline Recessive & & & & $1.57(1.29-1.84)$ & $<0.01$ \\
\hline G & $243(52.83)$ & $293(63.70)$ & & 1.00 (reference) & \\
\hline A & $217(47.17)$ & $167(36.30)$ & & $1.27(1.09-1.42)$ & 0.001 \\
\hline \multicolumn{6}{|l|}{ rs3741219 } \\
\hline AA & $124(53.91)$ & $115(50.00)$ & 0.04 & 1.00 (reference) & \\
\hline $\mathrm{AG}$ & 99 (43.04) & $104(45.22)$ & & $0.94(0.77-1.14)$ & 0.58 \\
\hline GG & $7(3.04)$ & $11(4.78)$ & & $0.75(0.35-1.26)$ & 0.42 \\
\hline Additive & & & & $0.96(0.83-1.13)$ & 0.70 \\
\hline Dominant & & & & $0.92(0.76-1.12)$ & 0.46 \\
\hline Recessive & & & & $0.77(0.36-1.28)$ & 0.47 \\
\hline A & 347 (75.43) & $334(72.61)$ & & 1.00 (reference) & \\
\hline $\mathrm{G}$ & $113(24.57)$ & $126(27.39)$ & & $0.93(0.79-1.08)$ & 0.37 \\
\hline \multicolumn{6}{|l|}{ rs7158663 } \\
\hline GG & $101(43.91)$ & $125(54.35)$ & 0.12 & 1.00 (reference) & \\
\hline GA & 85 (36.96) & $95(41.30)$ & & $1.06(0.84-1.32)$ & 0.68 \\
\hline $\mathrm{AA}$ & $44(19.13)$ & $10(4.35)$ & & $1.82(1.46-2.11)$ & $<0.01$ \\
\hline Additive & & & & $1.12(0.94-1.34)$ & 0.22 \\
\hline Dominant & & & & $1.23(1.02-1.50)$ & 0.03 \\
\hline Recessive & & & & $1.78(1.45-2.02)$ & $<0.01$ \\
\hline $\mathrm{G}$ & $287(62.39)$ & $345(75.00)$ & & 1.00 (reference) & \\
\hline A & $173(37.61)$ & $115(25.00)$ & & $1.32(1.16-1.50)$ & $<0.01$ \\
\hline
\end{tabular}

*Adjusted for age, gender, and BMI.

CI, confidence interval; HWE, Hardy-Weinberg equilibrium; OR, odds ratio. 
Table 3. Correlation between H19 Single Nucleotide Polymorphisms (SNPs) and MEG3 SNP and the Risk of Osteoarthritis in Different Age, Gender, and Body Mass Index Subpopulations

\begin{tabular}{|c|c|c|c|c|}
\hline & $O A(\mathrm{n}=230), \mathrm{n}(\%)$ & Control $(\mathrm{n}=230), \mathrm{n}(\%)$ & OR $(95 \% C I)^{*}$ & $\mathrm{p}^{*}$ \\
\hline \multicolumn{5}{|l|}{$\begin{array}{l}\text { Age, years } \\
<60\end{array}$} \\
\hline \multicolumn{5}{|l|}{ rs 217727} \\
\hline GG & $51(36.43)$ & 66 (40.49) & 1.00 (reference) & \\
\hline GA/AA & 89 (63.57) & $97(59.51)$ & $1.10(0.84-1.45)$ & 0.55 \\
\hline \multicolumn{5}{|l|}{$\mathrm{rs} 3741219$} \\
\hline AA & $74(52.86)$ & 77 (47.24) & 1.00 (reference) & \\
\hline AG/GG & $66(47.14)$ & $86(52.76)$ & $0.89(0.69-1.15)$ & 0.39 \\
\hline \multicolumn{5}{|l|}{ rs7158663 } \\
\hline GG & $61(43.57)$ & 85 (52.15) & 1.00 (reference) & \\
\hline GA/AA & 79 (56.43) & 78 (47.85) & $1.20(0.93-1.57)$ & 0.17 \\
\hline \multicolumn{5}{|l|}{$\geq 60$} \\
\hline \multicolumn{5}{|l|}{ rs217727 } \\
\hline GG & 33 (36.67) & 28 (41.79) & 1.00 (reference) & \\
\hline GA/AA & 57 (63.33) & $39(58.21)$ & $1.10(0.82-1.51)$ & 0.63 \\
\hline \multicolumn{5}{|l|}{ rs3741219 } \\
\hline AA & $50(55.56)$ & $38(56.72)$ & 1.00 (reference) & \\
\hline AG/GG & $40(44.44)$ & $29(43.28)$ & $1.02(0.76-1.36)$ & 0.99 \\
\hline \multicolumn{5}{|l|}{ rs7158663 } \\
\hline GG & $40(44.44)$ & $40(59.70)$ & 1.00 (reference) & \\
\hline GA/AA & $50(55.56)$ & $27(40.30)$ & $1.30(0.97-1.73)$ & 0.08 \\
\hline \multicolumn{5}{|l|}{ Gender } \\
\hline \multicolumn{5}{|l|}{ Men } \\
\hline \multicolumn{5}{|l|}{ rs 217727} \\
\hline GG & $34(38.20)$ & 34 (38.64) & 1.00 (reference) & \\
\hline GA/AA & $55(61.80)$ & $54(61.36)$ & $1.01(0.74-1.41)$ & 0.99 \\
\hline \multicolumn{5}{|l|}{ rs3741219 } \\
\hline AA & 49 (55.06) & 49 (55.68) & 1.00 (reference) & \\
\hline & $40(44.94)$ & $39(44.32)$ & $1.01(0.73-1.38)$ & 0.99 \\
\hline \multicolumn{5}{|l|}{ rs7158663 } \\
\hline GG & $29(32.58)$ & $41(46.59)$ & 1.00 (reference) & \\
\hline GA/AA & $60(67.42)$ & 47 (53.41) & $1.35(0.97-1.94)$ & 0.08 \\
\hline \multirow{2}{*}{\multicolumn{5}{|c|}{ Women }} \\
\hline & & & & \\
\hline GG & $50(35.46)$ & $60(42.25)$ & 1.00 (reference) & \\
\hline GA/AA & $91(64.54)$ & $82(57.75)$ & $1.16(0.90-1.52)$ & 0.29 \\
\hline \multicolumn{5}{|l|}{ rs3741219 } \\
\hline AA & 75 (53.19) & 66 (46.48) & 1.00 (reference) & \\
\hline AG/GG & $66(46.81)$ & $76(53.52)$ & $0.87(0.68-1.12)$ & 0.31 \\
\hline rs7158663 & & $10(35.02)$ & & \\
\hline GG & $72(51.06)$ & 84 (59.15) & 1.00 (reference) & \\
\hline GA/AA & 69 (48.94) & $58(40.85)$ & $1.18(0.92-1.50)$ & 0.21 \\
\hline BMI $\left(\mathrm{kg} / \mathrm{m}^{2}\right)$ & & & & \\
\hline$<24$ & & & & \\
\hline rs217727 & & & & \\
\hline GG & $14(34.15)$ & 17 (43.59) & 1.00 (reference) & \\
\hline GA/AA & $27(65.85)$ & $22(56.41)$ & $1.22(0.75-2.10)$ & 0.52 \\
\hline rs 3741219 & & & & \\
\hline AA & $26(63.41)$ & 19 (48.72) & 1.00 (reference) & \\
\hline AG/GG & $15(36.59)$ & $20(51.28)$ & $0.74(0.44-1.20)$ & 0.27 \\
\hline rs7158663 & & & & \\
\hline GG & $16(39.02)$ & $20(51.28)$ & 1.00 (reference) & \\
\hline GA/AA & $25(60.98)$ & 19 (48.72) & $1.28(0.79-2.12)$ & 0.38 \\
\hline$\geq 24$ & & & & \\
\hline rs 217727 & & & & \\
\hline GG & $70(37.04)$ & $77(40.31)$ & 1.00 (reference) & \\
\hline GA/AA & $119(62.96)$ & 114 (59.69) & $1.07(0.86-1.35)$ & 0.58 \\
\hline rs 3741219 & & & & \\
\hline AA & 98 (51.85) & $96(50.26)$ & 1.00 (reference) & \\
\hline AG/GG & $91(48.15)$ & 95 (49.74) & $0.97(0.78-1.20)$ & 0.84 \\
\hline rs 7158663 & & & & \\
\hline GG & 85 (44.97) & $105(54.97)$ & 1.00 (reference) & \\
\hline GA/AA & $104(55.03)$ & $86(45.03)$ & $1.22(0.99-1.52)$ & 0.07 \\
\hline
\end{tabular}

*Adjusted for age, gender, and BMI. 
Table 4. False Positive Report Rate Values of the Correlation Between Genotype and Allele Frequency OF RS217727 AND Rs3741219 LOCI OF H19 GENE AND Rs7158663 LOCUS OF MEG3 GENE AND RISK OF OsTEOARTHRITIS

\begin{tabular}{lcccc}
\hline & & & \multicolumn{2}{c}{ Prior probability } \\
\cline { 3 - 5 } Genotype & OR $(95 \%$ CI $)$ & Statistical power & 0.1 & 0.01 \\
\hline rs217727 AA vs. GG & $1.48(1.19-1.79)$ & 0.923 & 0.2332 & 0.7698 \\
rs217727 AA vs. GG+GA & $1.57(1.29-1.84)$ & 0.915 & 0.2228 & 0.7592 \\
rs217727 A vs. G & $1.27(1.09-1.42)$ & 0.899 & 0.2616 & 0.7958 \\
rs7158663 AA vs. GG & $1.82(1.46-2.11)$ & 0.923 & 0.1982 & 0.7312 \\
rs7158663 (GA+AA) vs. GG & $1.23(1.02-1.50)$ & 0.897 & 0.2679 & 0.8010 \\
rs7158663 AA vs. GG+GA & $1.78(1.45-2.02)$ & 0.932 & 0.2018 & 0.7355 \\
rs7158663 A vs. G & $1.32(1.16-1.50)$ & 0.894 & 0.2542 & 0.7895 \\
& & & 0.9656 \\
\end{tabular}

\section{Association of the H19 SNPS and MEG3 SNP with the risk of $O A$}

The genotype frequencies of the H19 SNPs rs217727 and the MEG3 SNP rs7158663 selected in this study were consistent with the Hardy-Weinberg equilibrium $(p>0.05$; Table 2). However, the genotype frequency of the rs 3741219 locus of the $H 19$ gene was not consistent with the HardyWeinberg equilibrium $(p=0.04)$. We observed a significant correlation between different genotypes and allele frequencies of H19 SNP rs 217727 and the risk of OA. The risk of OA in subjects carrying the AA genotype was 1.48 times higher compared with those carrying the GG genotype, after adjusting for age, gender, and BMI (95\% CI: 1.19-1.79, $p<0.01)$. The risk of OA was significantly increased, by 1.57 times, in the recessive model (95\% CI: $1.29-1.84, p<0.01$ ), whereas the risk of OA was not significantly increased for the GA genotype, additive model, or dominant model ( $p>0.05$; Table 2). The risk of OA was significantly increased in subjects carrying the A allele $(\mathrm{OR}=1.27,95 \% \mathrm{CI}$ : $1.09-1.42$, $p=0.001)$.

There was no significant change in the risk of OA between the AG and GG genotypes of the H19 SNP rs3741219 compared with that of the AA genotype $(p>0.05)$. There was no significant change in the risk of OA in the additive model, dominant model, or recessive model $(p>0.05)$. There was no significant difference in the risk of OA between subjects carrying the $\mathrm{G}$ allele and A allele $(p>0.05)$.

There was no significant difference in the risk of OA between subjects carrying the MEG3 SNP rs7158663 GA genotype compared with those carrying the GG genotype $(p>0.05)$. The risk of OA was not significantly increased in the additive model $(p>0.05)$. However, the risk of OA for those carrying the AA genotype was significantly increased, by 1.82 times, compared with that of GG genotype carriers (95\% CI: $1.46-2.11, p<0.01$ ), and the risk of OA was increased in both the dominant model $(\mathrm{OR}=1.23,95 \% \mathrm{CI}$ : $1.02-1.50, p=0.03)$ and the recessive model $(\mathrm{OR}=1.78,95 \%$ CI: $1.45-2.02, p<0.01)$. Furthermore, the risk of $\mathrm{OA}$ in subjects carrying the A allele was 1.32 times higher compared with those carrying the $\mathrm{G}$ allele (95\% CI: 1.16-1.50, $p<0.01$; Table 2).

\section{Stratified analyses of the correlations between $\mathrm{H} 19$ and MEG3 SNPS and the risk of OA}

We then divided the subjects into young (age $<60$ years) and elderly (age $\geq 60$ years), male and female, and nonobese
$\left(\mathrm{BMI}<24 \mathrm{~kg} / \mathrm{m}^{2}\right.$ ) and obese (BMI $\geq 24 \mathrm{~kg} / \mathrm{m}^{2}$ ) subgroups, and performed stratified analyses to determine the association of H19 and MEG3 SNPs with the risk of OA. The results showed that there was no significant difference in the association of the $H 19$ and MEG3 SNPs with the risk of OA between age, gender, and BMI subgroups $(p>0.05)$ (Table 3$)$.

\section{False-positive report rate}

The false-positive report rate (FPRP) values of correlation between genotype and allele frequency of $H 19$ gene rs 217727 and rs3741219 loci, and the MEG3 gene rs7158663 locus and the risk of OA are shown in Table 4. The rs7158663 AA genotype was significantly associated with OA susceptibility. When the prior probability was 0.1, FPRP was $<0.2$. The FPRP value of the correlation between genotypes and alleles of other SNP loci and susceptibility to OA was $>0.2$, indicating that the sample size in this study was small, which affected the objectivity of the analysis of rs7158663 AA genotype and OA susceptibility. Thus, there may be bias, which needs to be further verified in a large sample (He et al., 2013).

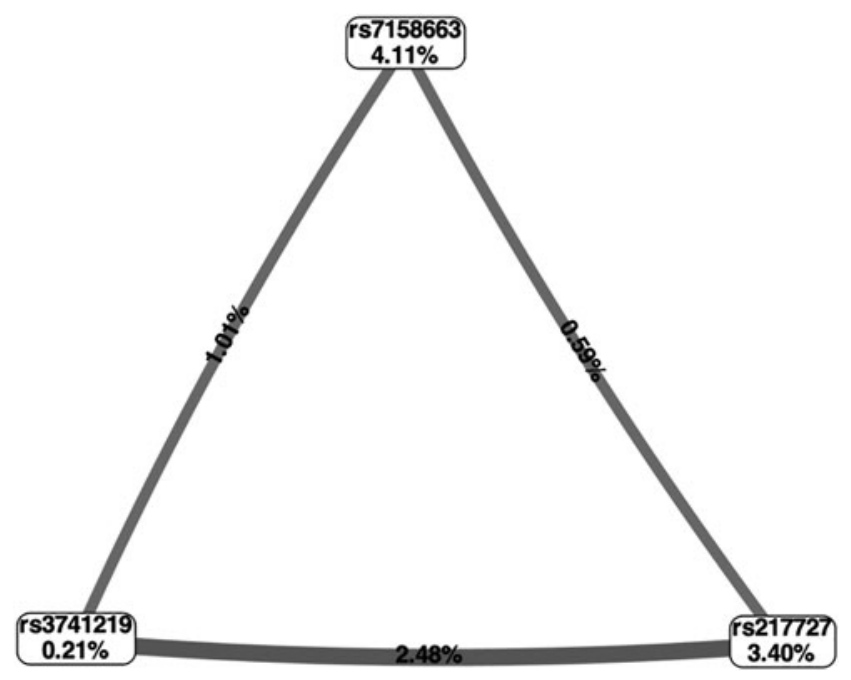

FIG. 1. Multifactor dimensionality reduction analysis of the interaction between the H19 SNP loci and the MEG3 SNP locus. A larger number on the line indicates a stronger interaction between the two SNP loci. A larger number under the SNP locus indicates a greater risk of OA occurrence. MEG3, maternal expressed gene 3; OA, osteoarthritis; SNP, single nucleotide polymorphism. 


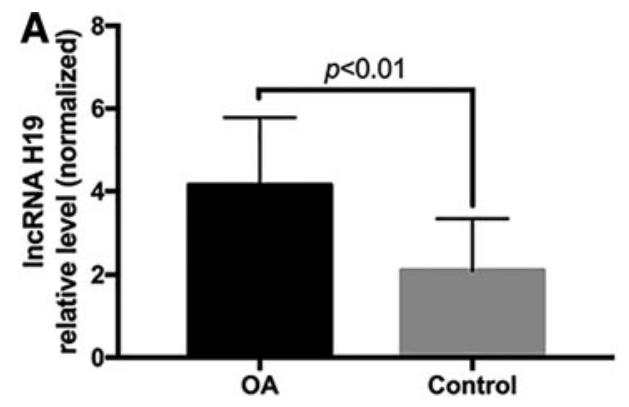

Multifactor dimensionality reduction analysis of the interaction between $\mathrm{H} 19$ and MEG3 SNPs

Multifactor dimensionality reduction was performed to analyze the interaction among $H 19$ SNPs rs217727 and rs3741219 SNPs and MEG3 SNP rs7158663. The interaction between SNPs rs3741219 and rs217727 was the strongest $(2.48 \%)$, followed by that between SNP rs3741219 and SNP rs7158663 (1.01\%), with the lowest interaction between SNP rs217727 and SNP rs7158663 (0.59\%; Fig. 1).

\section{Upregulation of IncRNA H19 levels and downregulation of IncRNA MEG3 levels in the plasma of patients with $O A$}

We used quantitative real-time reverse transcription-PCR (qRT-PCR) to analyze the expression plasma levels of lncRNAs H19 and MEG3. The lncRNA H19 level in the plasma of patients suffering from OA was significantly higher compared with the control group, and the plasma lncRNA $M E G 3$ level was significantly lower compared with the control group $(p<0.01$; Fig. $2 \mathrm{~A}, \mathrm{~B})$.

\section{Comparison of plasma miRNA levels between patients with $O A$ and controls}

We used qRT-PCR to analyze plasma hsa-miR-4804-5p, hsa-miR-8071, hsa-miR-8072, hsa-miR-3960, hsa-miR4307, and hsa-miR-1265 levels. In the patients with OA, the plasma levels of hsa-miR-4804-5p, hsa-miR-8071, hsa-miR8072, and hsa-miR-3960 were significantly lower than those in the control group, while the levels of hsa-miR-8072 and hsa-miR-3960 were significantly higher than those in the control group ( $p<0.05$; Fig. 3$)$.

\section{Correlations between IncRNA H19 and MEG3 levels and miRNA levels in plasma}

Pearson correlation analysis showed that the plasma lncRNA H19 level correlated negatively with the levels of hsa-miR-4804-5p, hsa-miR-8071, hsa-miR-8072, and hsamiR-3960 $\left(r^{2}=0.5662,0.3582,0.5001\right.$, and 0.5864 , respectively; $p<0.001)$, and the lncRNA $M E G 3$ level correlated negatively with the levels of hsa-miR-4307 and hsa-miR1265 ( $r^{2}=0.4173$ and 0.2633 , respectively; $p<0.001$; Fig. 4).

\section{SNP rs217727 of $\mathrm{H} 19$ correlation with plasma IncRNA H19 level}

The level of lncRNA H19 in the plasma of those carrying the A allele (GA/AA) of H19 SNP rs217727 was significantly higher compared with those carrying the GG genotype $(p<0.01)$. However, there was no significant difference in the plasma lncRNA $H 19$ level between subjects with different genotypes of the rs3741219 locus ( $p=0.10$; Fig. 5).

\section{Correlation between $\mathrm{H} 19$ SNP rs217727 and plasma miRNAs levels}

The plasma levels of hsa-miR-4804-5p, hsa-miR-8071, hsamiR-8072, and hsa-miR-3960 in subjects carrying the A allele (GA/AA) of H19 SNP rs217727 were significantly lower compared with subjects carrying the GG allele $(p<0.05$; Fig. 6A-D). However, there were no significant differences in the plasma levels of hs-miR-4804-5p, hsa-miR-8071, hsamiR-8072, and hsa-miR-3960 in subjects with different genotypes of the rs3741219 locus ( $p>0.05$; Fig. 6E).

\section{Correlation between MEG3 SNP rs7158663 and plasma miRNAs levels}

The plasma levels of lncRNA MEG3 in subjects carrying the A allele (GA/AA) of MEG3 SNP rs7158663 were significantly lower than those of subjects carrying the GG genotype (Fig. 7A). However, the plasma levels of hsa-miR4307 and hsa-miR-1265 were significantly higher than those of subjects with the GG genotype $(p<0.001$; Fig. 7B, C).

\section{Discussion}

The present study demonstrates that lncRNA H19 SNP rs217727 and lncRNA MEG3 SNP rs7158663 are associated

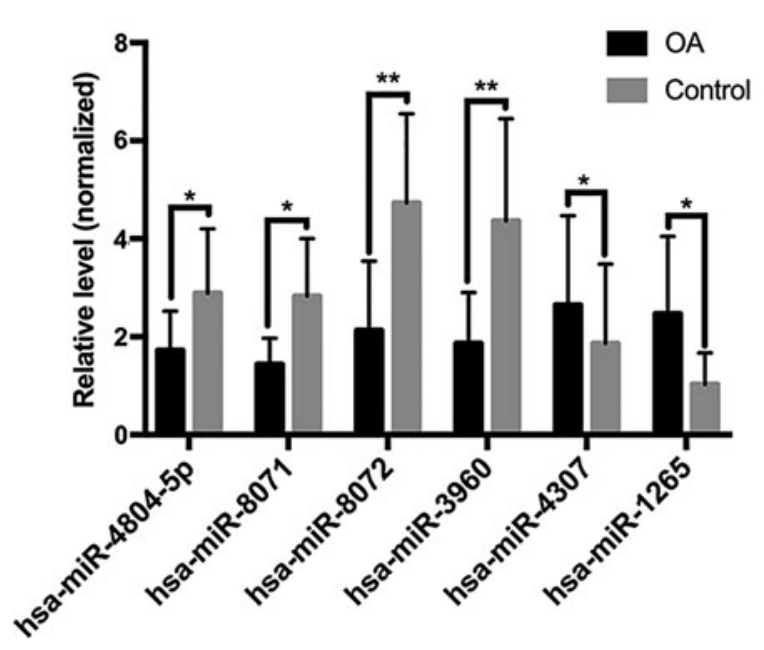

FIG. 3. Comparison of plasma miRNA levels between patients with OA and controls. ${ }^{*} p<0.05$; $* * p<0.01$, compared with that of the control group. miRNA, microRNA. 

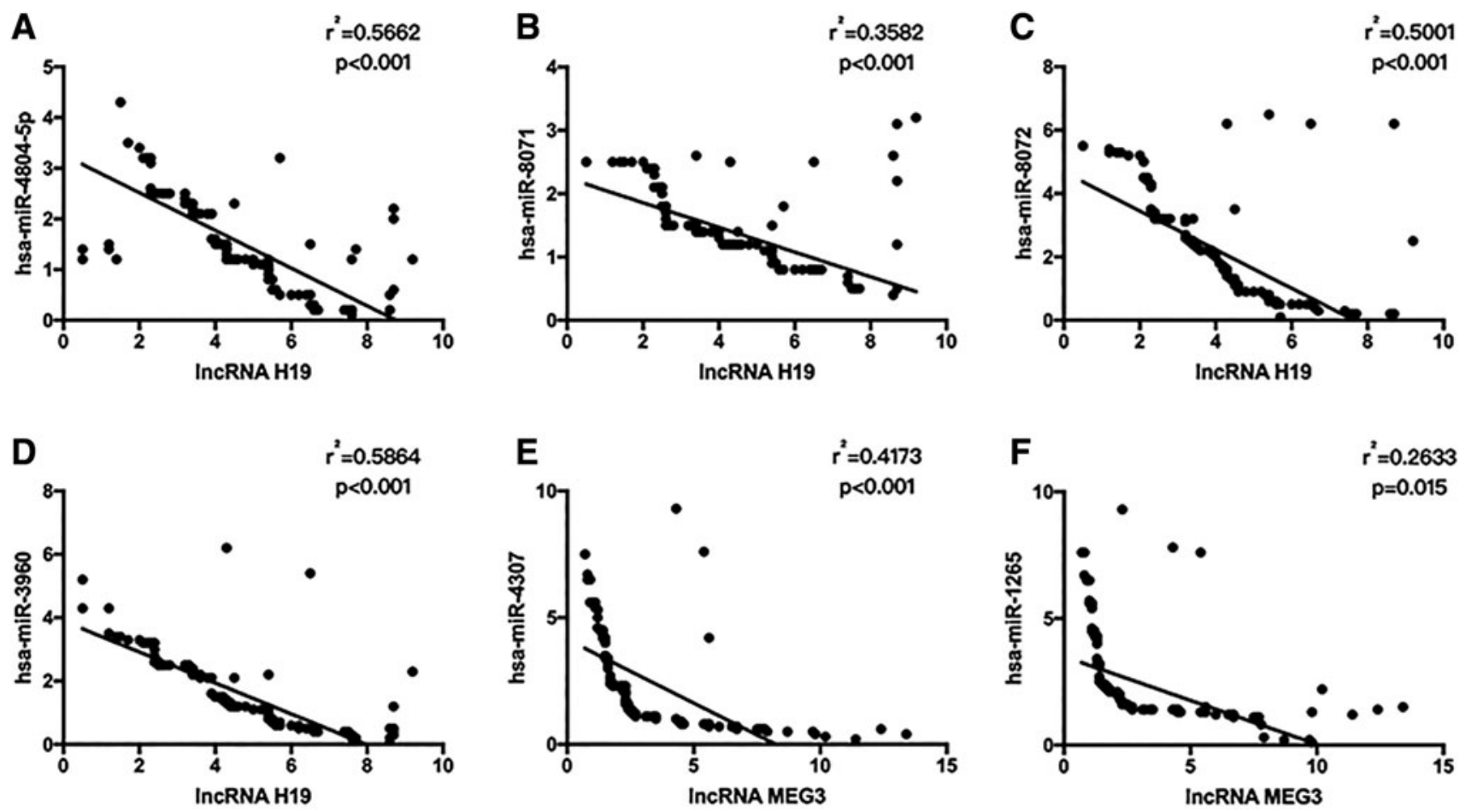

FIG. 4. Correlation between plasma lncRNA and miRNA levels in patients with OA. (A) Comparison of the lncRNA $H 19$ level with the hsa-miR-4804-5p level. (B) Comparison of the lncRNA H19 level with the hsa-miR-8071 level. (C) Comparison of the lncRNA H19 level with the hsa-miR-8072 level. (D) Comparison of the lncRNA H19 level with the hsa-miR-3960 level. (E) Comparison of the lncRNA MEG3 level with the hsa-miR-4307 level. (F) Comparison of the lncRNA MEG3 level with the hsa-miR-1265 level.

with the risk of $\mathrm{OA}$ in a Han Chinese population. The findings of the analyses of plasma miRNAs levels and lncRNA $H 19$ and MEG3 levels support the speculation that H19 SNP rs217727 influences the expression levels of lncRNA H19, hsa-miR-4804-5p, hsa-miR-8071, hsa-miR-8072, and hsa-miR-3960, whereas MEG3 SNP rs7158663 affects the expression levels of IncRNA MEG3, hsa-miR-4307, and hsamiR-1265. However, there is no direct evidence supporting this hypothesis in the present study.

$\mathrm{OA}$ is a common arthropathy in the elderly, which has become a major health problem in this age group (Poulet and Staines, 2016; Chen et al., 2017). The mechanism of OA occurrence is not fully understood. To develop better therapeutic approaches for $\mathrm{OA}$, it is necessary to determine its underlying mechanisms. Recent studies have shown that IncRNAs play a key role in the occurrence and development of OA, including lncRNA H19 (Steck et al., 2012; Wang et al., 2018; Hu et al., 2019) and lncRNA MEG3 (Su et al., 2015). An elegant study demonstrated that up to 73 lncRNAs are upregulated in chondrocytes from patients with OA, including lncRNA H19 (Xing et al., 2014). In the present study, lncRNA H19 expression was upregulated in the plasma of patients with OA, indicating that in these patients, the expression level of lncRNA H19 was significantly increased in chondrocytes as well as in plasma. MEG3 is a maternally expressed lncRNA that is closely related to inflammationrelated diseases, including OA (Su et al., 2015; Li et al., 2018). Recent studies showed that MEG3 is downregulated in
FIG. 5. Correlation between plasma lncRNA $H 19$ levels and H19 SNPs in patients with OA. (A) The plasma lncRNA H19 levels in different genotypes of the H19 SNP rs217727. (B) The same as in (A) for SNP rs3741219.
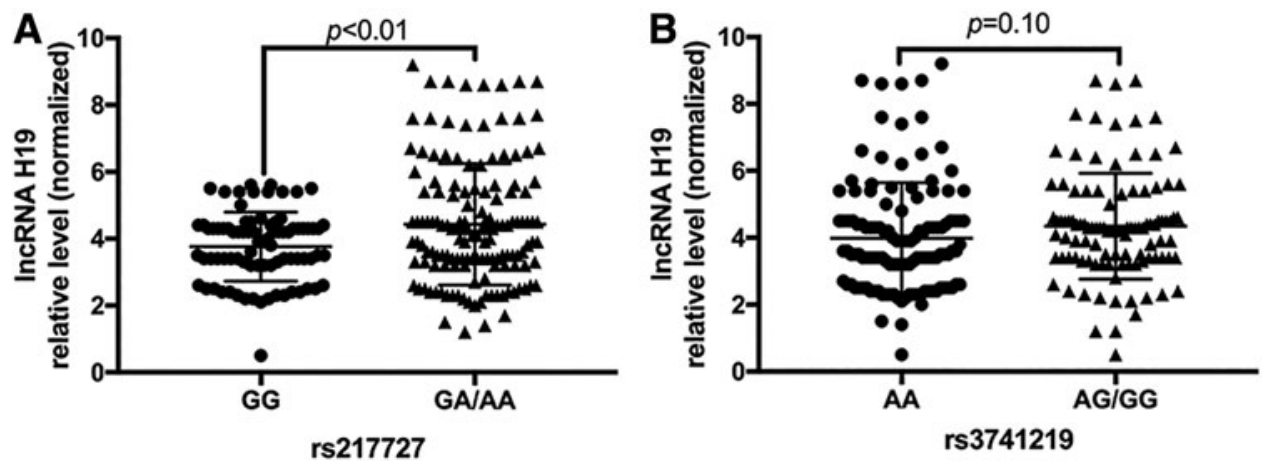

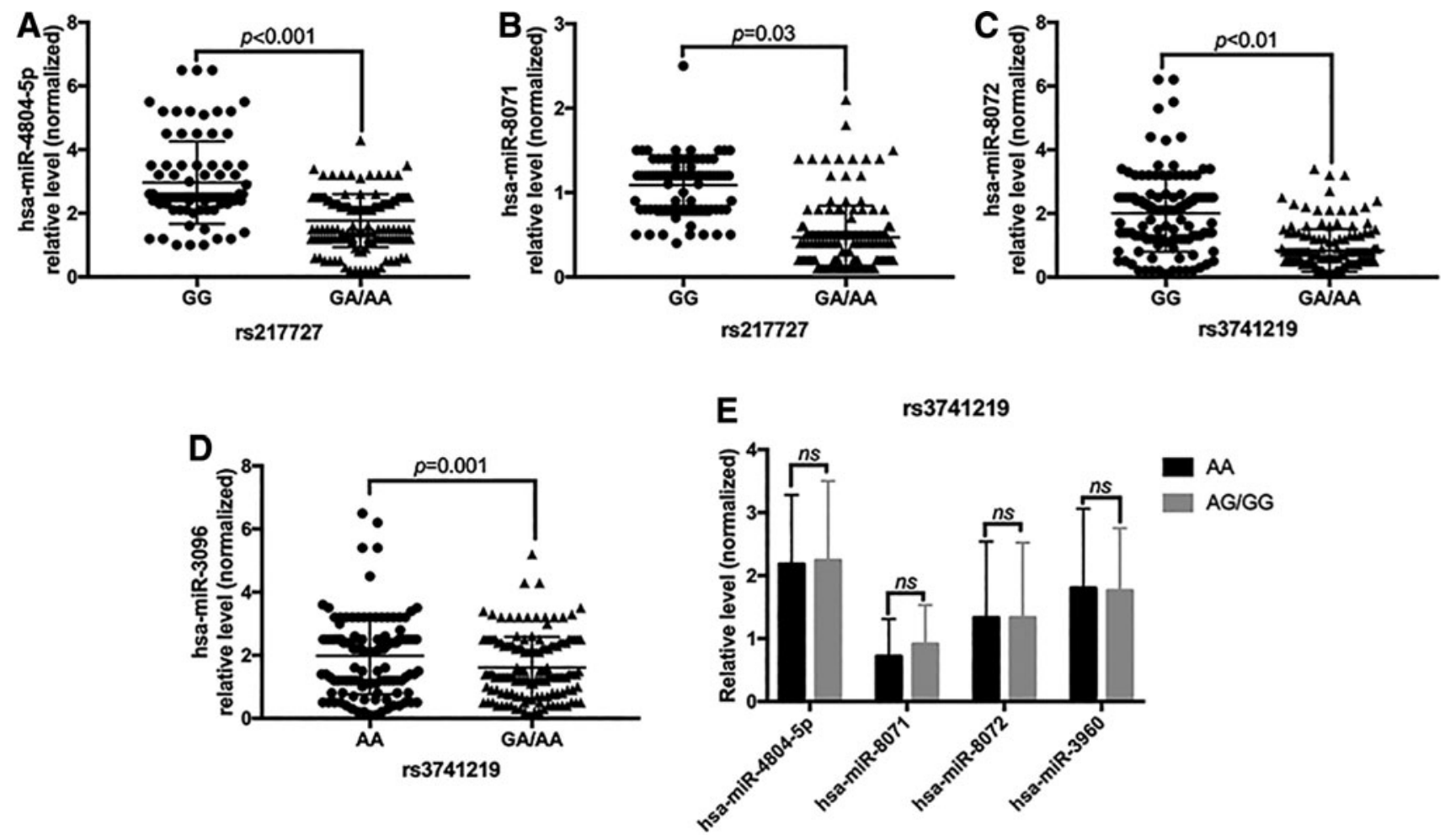

FIG. 6. Correlation between plasma miRNA levels and H19 SNPs in patients with OA. (A) Comparison of plasma hsamiR-4804-5p level in patients with OA carrying different genotypes of the rs217727 locus. (B) The same analysis as in (A) for hsa-miR-8071. (C) The same analysis as in (A) for hsa-miR-8072. (D) The same analysis as in (A) for hsa-miR3096. (E) Comparison of the plasma levels of hsa-miR-4804-5p, hsa-miR-8071, hsa-miR-8072, and hsa-miR-3960 in patients with OA carrying different genotypes of the rs3741219 locus. ns, no significant difference.

OA tissues, and might participate in the development and progression of OA by inhibiting the expression of vascular endothelial growth factor (Su et al., 2015). We also found that the expression level of IncRNA $M E G 3$ was downregulated in the plasma of patients with OA, indicating that IncRNA $M E G 3$ is a potential target molecule to diagnose and treat OA.

Our case-control study demonstrated that the A allele of the $H 19$ SNP rs217727 and the A allele of MEG3 SNP rs7158663 are high risk factors for OA. In subjects carrying A allele of $H 19$ SNP rs 217727 , the risk of OA was increased by
1.27 times, and in subjects carrying the A allele of $M E G 3$ SNP rs7158663, the risk of OA was increased by 1.32 times, after adjusting for age, gender, BMI, and other factors. These results indicate that $H 19$ gene rs 217727 locus and MEG3 gene rs7158663 locus SNPs are significantly related to the risk of OA. Further analyses revealed that SNPs at the H19 rs217727 locus and the MEG3 rs7158663 locus altered the expression levels of IncRNA H19 and lncRNA MEG3, respectively. Using the online IncRNA-miRNA interaction prediction tool, we predicted that lncRNA H19 could target hsa-miR-4804-5p, hsa-miR-8071, hsa-miR-8072, and hsa-
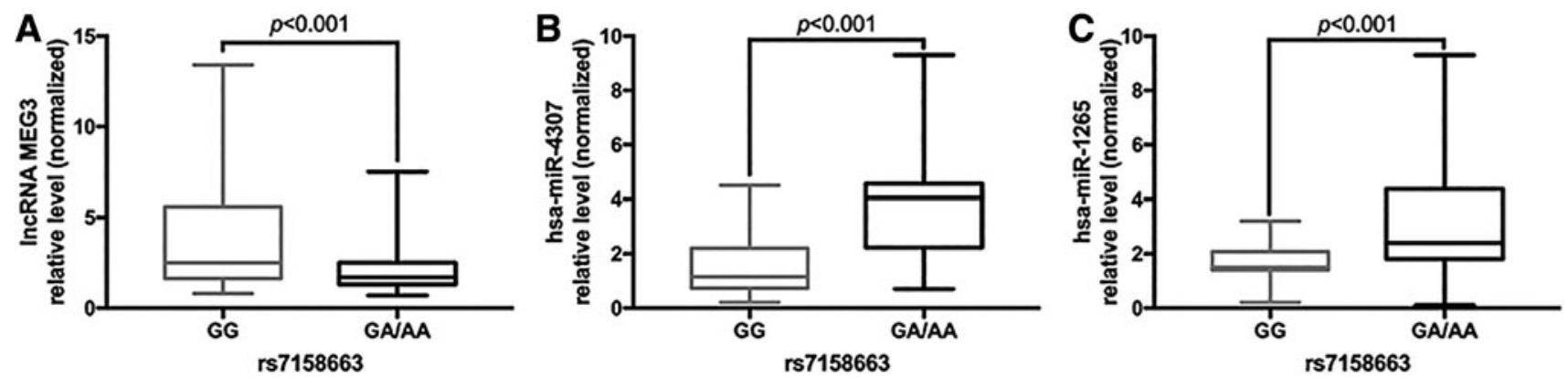

FIG. 7. Correlation between the MEG3 SNP, plasma lncRNA, and miRNA levels in patients with OA. (A) The level of lncRNA MEG3 in the plasma of subjects carrying the A allele (GA/AA) of MEG3 SNP rs7158663 was significantly reduced. (B) The level of hsa-miR-4307 in the plasma of subjects carrying the A allele (GA/AA) of MEG3 SNP rs7158663 was significantly increased. (C) The same analysis as in (B) for hsa-miR-1265. 
miR-3960. Furthermore, lncRNA MEG3 could target hsamiR-4307 and hsa-miR-1265. More importantly, locus rs217727 is located at the binding site of hsa-miR-4804-5p, hsa-miR-8071, hsa-miR-8072, and hsa-miR-3960, and locus rs7158663 is located at the binding site of hsa-miR-4307 and hsa-miR-1265.

The results of the present study indicated a negative correlation between the level of lncRNA H19 and hsa-miR-48045p, hsa-miR-8071, hsa-miR-8072, and hsa-miR-3960 levels. Similarly, the lncRNA MEG3 level correlated negatively with the levels of hsa-miR-4307 and hsa-miR-1265. Therefore, we speculate that lncRNA H19 downregulates the levels of hsamiR-4804-5p, hsa-miR-8071, hsa-miR-8072, and hsa-miR3960. Furthermore, we suggest that 1ncRNA MEG3 reduces the levels of hsa-miR-4307 and hsa-miR-1265.

H19 SNP rs217727 was associated with the plasma levels of lncRNA H19, hsa-miR-4804-5p, hsa-miR-8071, hsa-miR8072, and hsa-miR-3960, while MEG3 SNP rs7158663 was associated with the plasma levels of lncRNA $M E G 3$, hsa-miR4307, and hsa-miR-1265. Therefore, it is likely that SNPs rs217727 and rs7158663 alter the binding efficiency of these lncRNAs to their target miRNAs, which in turn would affect the regulation of target mRNAs by the miRNAs.

There was no direct evidence showing that lncRNA H19 negatively regulates hsa-miR-4804-5p, hsa-miR-8071, hsamiR-8072, and hsa-miR-3960, or that IncRNA MEG3 downregulates hsa-miR-4307 and hsa-miR-1265. We speculate that lncRNA H19 may serve as a molecular sponge of hsa-miR-4804-5p, hsa-miR-8071, hsa-miR-8072, and hsamiR-3960, and that lncRNA MEG3 may serve a similar role concerning hsa-miR-4307 and hsa-miR-1265. These speculations have not been verified in cell models, which is a limitation of this study. Future in vitro and in vivo studies are needed to confirm these results. Additionally, we found that SNP rs3741219 is not associated with the risk of OA, although this SNP locus is also located at the miRNA-binding site of lncRNA H19. However, the genotype frequency distribution of rs3741219 locus in the control group selected in this study was not consistent with the Hardy-Weinberg equilibrium $(p=0.04)$. This result might have been affected by the relatively small sample size. Thus, the results should be verified using a larger sample. The analysis of the FPRP showed that the small sample size in this study affected the objectivity of analysis results of the rs7158663 AA genotype and OA susceptibility. The analysis may be biased and need to be further verified in a large sample size Moreover, we could not exclude the possibility of other SNPs and miRNAs that might be involved in the development of OA. Further studies will be required to better elucidate the role of IncRNAs and miRNAs in the development and progression of OA. Finally, the differential expression levels of related inflammatory factors in synovial fluid and whether there is a certain relationship between lncRNAs and microRNAs identified in this study and the differentially expressed inflammatory factors also need further research and exploration to further clarify its mechanism of occurrence and development of OA.

In summary, H19 SNP rs217727 and the MEG3 SNP rs7158663 are associated with the risk of OA. Further in vitro studies are necessary to confirm the underlying molecular mechanism.

\section{Author Disclosure Statement}

The authors declare no conflict of interest.

\section{Funding Information}

This work was supported by a grant from the Medical Health Science and Technology Program of Zhejiang Province (2020KY676).

\section{References}

Chen D, Shen J, Zhao W, et al. (2017) Osteoarthritis: toward a comprehensive understanding of pathological mechanism. Bone Res 5:16044.

Deane KD, Demoruelle MK, Kelmenson LB, et al. (2017) Genetic and environmental risk factors for rheumatoid arthritis. Best Pract Res Clin Rheumatol 31:3-18.

Fu M, Huang G, Zhang Z, et al. (2015) Expression profile of long noncoding RNAs in cartilage from knee osteoarthritis patients. Osteoarthritis Cartilage 23:423-432.

Ghaedi H, Zare A, Omrani MD, et al. (2018) Genetic variants in long noncoding RNA H19 and MEG3 confer risk of type 2 diabetes in an Iranian population. Gene 675:265-271.

He J, Wang MY, Qiu LX, et al. (2013) Genetic variations of mTORC1 genes and risk of gastric cancer in an Eastern Chinese population. Mol Carcinog 52(Suppl. 1):E70-E79.

Herman A, Chechik O, Segal G, et al. (2015) The correlation between radiographic knee OA and clinical symptoms-do we know everything? Clin Rheumatol 34:1955-1960.

Hu Y, Li S, Zou Y (2019) Knockdown of LncRNA H19 relieves LPS-induced damage by modulating miR-130a in osteoarthritis. Yonsei Med J 60:381-388.

Kellgren JH, Lawrence JS (1957) Radiological assessment of osteo-arthrosis. Ann Rheum Dis 16:494-502.

Kim H, Sung YK, Choi S, et al. (2019) Increased risk of malignancy in patients aged over 50 with idiopathic inflammatory myositis compared to patients with osteoarthritis of the knee. Mod Rheumatol 18:1-8.

Lane NE, Shidara K, Wise BL (2017) Osteoarthritis year in review 2016: clinical. Osteoarthritis Cartilage 25:209-215.

Li X, Tang C, Wang J, et al. (2018) Methylene blue relieves the development of osteoarthritis by upregulating lncRNA MEG3. Exp Ther Med 15:3856-3864.

Li Z, Niu Y (2019) Association between lncRNA H19 (rs217727, rs2735971 and rs3024270) polymorphisms and the risk of bladder cancer in Chinese population. Minerva Urol Nefrol 71:161-167.

Liao J, Yu X, Hu X, et al. (2017) lncRNA H19 mediates BMP9induced osteogenic differentiation of mesenchymal stem cells (MSCs) through Notch signaling. Oncotarget 8:5358153601.

Naili JE, Brostrom EW, Clausen B, et al. (2019) Measures of knee and gait function and radiographic severity of knee osteoarthritis-a cross-sectional study. Gait Posture 74: 20-26.

Ponting CP, Oliver PL, Reik W (2009) Evolution and functions of long noncoding RNAs. Cell 136:629-641.

Poulet B, Staines KA (2016) New developments in osteoarthritis and cartilage biology. Curr Opin Pharmacol 28:8-13.

Schuring N, Aoki H, Gray J, et al. (2017) Osteoarthritis is associated with symptoms of common mental disorders among former elite athletes. Knee Surg Sports Traumatol Arthrosc 25:3179-3185. 
Steck E, Boeuf S, Gabler J, et al. (2012) Regulation of H19 and its encoded microRNA-675 in osteoarthritis and under anabolic and catabolic in vitro conditions. J Mol Med (Berl) 90: 1185-1195.

Su W, Xie W, Shang Q, et al. (2015) The long noncoding RNA MEG3 is downregulated and inversely associated with VEGF levels in osteoarthritis. Biomed Res Int 2015: 356893.

Traylor M, Curtis C, Patel H, et al. (2017) Genetic and environmental risk factors for rheumatoid arthritis in a UK African ancestry population: the GENRA case-control study. Rheumatology (Oxford) 56:1282-1292.

Wang K, Chu M, Ding W, et al. (2018) Putative functional variants of lncRNA identified by RegulomeDB were associated with knee osteoarthritis susceptibility. BMC Musculoskelet Disord 19:284.

Xiang YJ, Dai SM (2009) Prevalence of rheumatic diseases and disability in China. Rheumatol Int 29:481-490.
Xing D, Liang JQ, Li Y, et al. (2014) Identification of long noncoding RNA associated with osteoarthritis in humans. Orthop Surg 6:288-293.

$\mathrm{Xu}$ J, Xu Y (2017) The lncRNA MEG3 downregulation leads to osteoarthritis progression via miR-16/SMAD7 axis. Cell Biosci 7:69.

Address correspondence to: Jun Yao, $M D$

Department of Orthopedics Hangzhou Dingqiao Hospital (Hangzhou Hospital of Traditional Chinese Medicine, Dingqiao District) 1630 Huanding Road Jianggan, Hangzhou, Zhejiang, 310005

China

E-mail: yj417407100@163.com 